\title{
IDENTIFICATION OF SSR MARKERS FORHYBRIDITY AND SEED GENETIC PURITY TESTING IN SUNFLOWER (Helianthus annuus L.)
}

\author{
Pallavi, H.M. ${ }^{*} 1$, Rame Gowda ${ }^{1}$, Shadakshari, Y.G. ${ }^{2}$, \\ Bhanuprakash, K. ${ }^{3}$ and Vishwanath, K. ${ }^{1}$ \\ ${ }^{1}$ Department of Seed Science \& Technology, University of Agricultural Sciences, \\ Bangalore 560065, India \\ ${ }^{2}$ All India Coordinated Crop Improvement Project on Sunflower, \\ University of Agricultural Sciences, Bangalore 560065, India \\ ${ }^{3}$ Seed Science and Technology Section, Indian Institute of Horticultural Research, \\ Hessaragatta, Bangalore 560089, India
}

Received: August 12, 2010

Accepted: January 25, 2011

\section{SUMMARY}

The genuineness of a hybrid is one of the most important characteristics of good quality seed. In order to identify pure hybrid and pollen shedders/ offtypes an investigation was performed to identify an ideal SSR marker. 58 primer pairs were screened to identify the specific marker associated with each hybrid and parental lines. Hybrid KBSH-44 could be clearly identified by using ORS 309 and ORS 170, based on the banding pattern resolved on polyacrylamide gel $(6 \%)$. The complementary banding pattern of both parents made a way to identify the hybrid. ORS 309 amplified allele size at $250 \mathrm{bp}$ was specific to female parent (CMS-17A) and 230 bp was specific to male parent (RHA 95C-1). These two bands of allele size 230 and $250 \mathrm{bp}$ were found only in hybrid KBSH-44. Another SSR primer ORS 170 was able to distinguish the hybrid KBSH-44 by amplifying allele of size 230 bp a female specific (CMS-17A) allele and $200 \mathrm{bp}$ amplicon a male specific allele (RHA 95-C-1). SSR primer ORS 811 found specific to identify KBSH-53 and it amplified allele of size $270 \mathrm{bp}$ in its female parent (CMS-53A) and allele size of $230 \mathrm{bp}$ in its pollen parent (RHA 95-C-1). The hybrid has both the alleles from its parents at 270 and $230 \mathrm{bp}$.

Key words: cms lines, genetic purity, sunflower (Helianthus annuus), Grow Out Test (GOT), hybrids, SSR marker

\section{INTRODUCTION}

The sunflower (Helianthus annuus L.) is one of the major annual world crops grown for edible oil and popular for its PUFA, vitamin $\mathrm{E}$ and is easy to refine. The increased production and productivity is credited to the release of new high yielding

* Corresponding author: Phone: 919844830858, e-mail: pallavihm@gmail.com 
varieties and hybrids for commercial cultivation. Higher genetic purity is an essential prerequisite for the commercialization of any hybrid seeds. Besides, success of any hybrid technology depends on the availability of quality seed supplied in time at reasonable cost. The genetic purity during multiplication stages is prone to contaminate due to the presence of pollen shedders, out crossing with foreign pollens etc., besides physical admixtures. Thus use of seeds with low genetic purity results in segregation of the traits, lower yields and genetic deterioration of varieties. Genetic purity test is done to verify any deviation from genuineness of the variety during its multiplications. For certification, genetic purity test is compulsory for all foundation and certified hybrid seeds.

The traditional Grow out Test (GOT) is done to determine the seed genetic purity test based on morphological markers are time consuming and are environmental dependence. To overcome this disadvantage, the biochemical markers are being used in many crops. However, repeatability and accuracy of these results on biochemical markers are subject to question. This made a way for the use of DNA molecular markers particularly the co-dominant markers. The SSR markers are of great importance for rapid assessment of hybrid and parental line seed purity (Yashitola et al., 2002 and Sundaram et al., 2008). In sunflower, a set of SSR markers have been identified to distinguish inbreed lines (Solodenko et al., 2003 and Antonova et al., 2006). However, the SSR markers require identification of heterozygosity of the sunflower hybrids and distinguishing the hybrid in question from its parental lines and other hybrids. Therefore, the present study was conducted to identify a specific SSR marker to discriminate the sunflower hybrids form its parental lines.

\section{MATERIAL AND METHODS}

The study included five hybrids, four female lines and two male lines which are listed in Table 1. Pure seeds of these hybrids and their parental lines were obtained from the All India Coordinated Crop Improvement Project on Sunflower, University of Agricultural Sciences, GKVK, Bangalore 560065. The seeds were cleaned, dried to the safe level of moisture $(<9 \%)$ and preserved for further usage.

Table 1: List of sunflower hybrids and their parental lines used in the study

\begin{tabular}{lccc}
\hline Hybrid & Seed parent (A line) & Maintainer line (B line) & Pollen parent (R line) \\
\hline KBSH-1 & CMS 234A & CMS 234B & RHA 6D-1 \\
KBSH- 41 & CMS 234A & CMS 234B & RHA 95 C-1 \\
KBSH-42 & CMS 851A & CMS 851B & RHA 95 C-1 \\
KBSH-44 & CMS 17A & CMS 17B & RHA 95 C-1 \\
KBSH-53 & CMS 53 A & CMS 53B & RHA 95 C-1 \\
\hline
\end{tabular}

DNA was extracted using CTAB protocol. About $0.1 \mathrm{~g}$ of young leaf tissue from each sample was homogenized in liquid nitrogen and incubated at $60^{\circ} \mathrm{C}$ for $30-45$ 
min with $500 \mu \mathrm{l}$ of CTAB buffer (1.0 M pH 8.0 Tris-HCl, $3 \mathrm{ml} \mathrm{NaCl,} 0.5$ EDTA, 1\% PVP-360). Then $500 \mu \mathrm{l}$ of chloroform: isoamyl alcohol mixture (24:1) was added and blended thoroughly. After centrifugation (5 min, 13,000 rpm), supernatant layer was pipette out into a new eppendorf tube and an approximately equal volume of chilled ethanol was added. After storage at $-20^{\circ} \mathrm{C}$ for $30-60 \mathrm{~min}$, precipitated DNA was centrifuged, vacuum dried and finally stored in TE buffer.

\section{PCR amplification}

Fifty eight SSR primer pairs were used in the study. The volume of the reaction mixture was $12 \mu \mathrm{l}$. It consists of $30 \mathrm{ng}$ of template DNA, $1 \times$ PCR buffer with 1.5 $\mathrm{mM}$ of $\mathrm{MgCl}_{2}, 0.2 \mathrm{mM}$ of dNTPs, $0.25 \mu \mathrm{M}$ each of forward and reverse primers and $1 \mathrm{U}$ of Taq DNA polymerase. Thermal Cycler was used and programmed for 30 cycles of $94^{\circ} \mathrm{C}(3 \mathrm{~min}), 50-55^{\circ} \mathrm{C}\left(40 \mathrm{sec}\right.$ ), $72^{\circ} \mathrm{C}$ ( $40 \mathrm{sec}$ ), then followed by finalextension at $72^{\circ} \mathrm{C}$ for $10 \mathrm{~min}$. PCR products (7.0-7.5 $\left.\mu \mathrm{l}\right)$ were used for electrophoresis on six percent acryl amide gels stained with ethidium bromide at $100-120 \mathrm{~V} / \mathrm{cm}$ for 100-200 min and photographed using documentation unit under UV light.

Analysis of DNA banding pattern was carried on Statistica Package. The program used was joining cluster analysis (tree clustering) with raw input data of each population separately. The main parameter which guided the joining cluster process linkage rule was Unweighed Pair Grouping Average (UPGMA) and the distance was computed from raw data using squared Euclidean distances and dendrogram was obtained.

\section{RESULTS AND DISCUSSION}

Characterization and identification of cultivars are crucial to varietal improvement, release and in seed production programme. It is mandatory to maintain the genetic purity of hybrid seed for the successful crop production. Unequivocal of characteristic pattern of hybrids can be obtained using DNA markers and had been termed as DNA fingerprinting. The use of DNA markers to obtain genotype specific profiles has distinct advantages over morphological and biochemical methods. The morphological markers are influenced by the environmental conditions, labour intensive time consuming and cumbersome. However, the biochemical markers such as isozyme and protein profiles are least influenced by the environment but exhibit limited polymorphism and often do not allow discrimination between closely related inbred lines (Lucchese et al., 1999). However, the DNA markers overcome most of these disadvantages of morphological and biochemical markers that can be useful to distinguish hybrids, its parental lines and off-types. The usefulness of DNA fingerprinting technique for cultivar identification was demonstrated by Dallas (1988) for the first time in rice.

The present study utilized the SSR marker technique for identification of five sunflower hybrids along with its parental lines (Table 1), and it is proved that this 
technique can be successfully applied to distinguish and identify the hybrids from their parental lines. Besides, SSR had much more polymorphism than most other DNA markers and is co-dominant and larger in quantity. Therefore, the high polymorphic information content (PIC) of SSR had promoted the application of microsatellites as molecular markers in fingerprinting (Ashikawa et al., 1999) of crop varieties.

In this study, 58 primer pairs of SSR's primer associated with each hybrid and parental lines were assessed on 3.5 percent agarose. Although the PCR products of the DNA samples on the agarose did appear, they failed to show any polymorphism among the hybrids and their parental lines. Probably the differences in the base pair sizes are too small to resolve and identify on the agarose. The primers which were not able to resolve and show polymorphism in agarose gel were found clearly resolving in polyacrylamide gel of six per cent.

The dendrogram of five hybrids and 6 parental lines were constructed based on the scored SSR marker banding pattern (Figure 1). Initially the cluster was divided into two major groups at 78 percent dissimilarity level. The upper cluster consisted of hybrids viz., KBSH-1, KBSH-41, KBSH-42, KBSH-44 and KBSH-53. The second cluster consisted of all parental lines ( $\mathrm{cms}$ lines and restorer lines) under study.

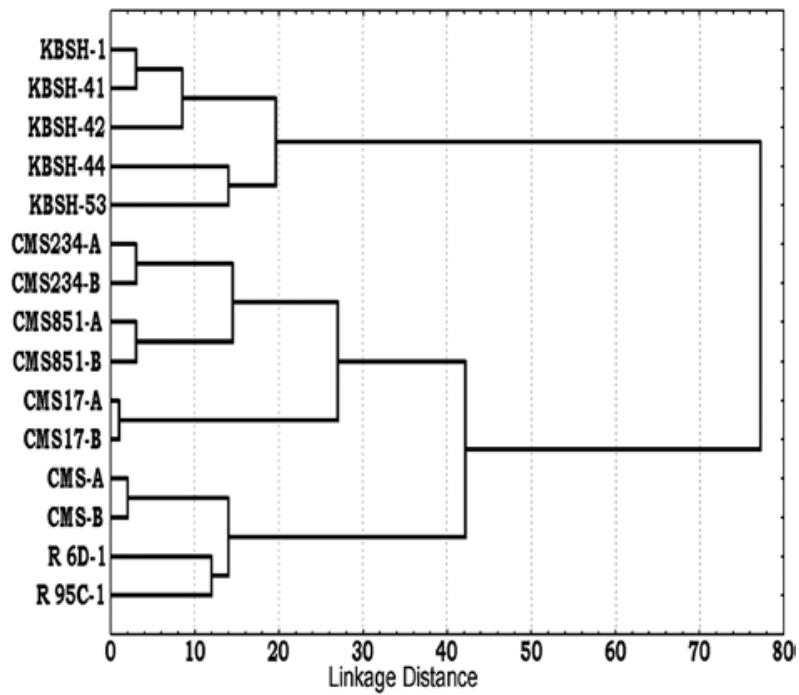

Figure 1: Dendrogram of sunflower hybrids and their parental lines based on SSR markers using UPGMA analysis (Squared Euclidean distances)

Further, the upper major cluster was grouped into three sub-clusters pooling KBSH-1 and KBSH-41 under one cluster, because they share the cytoplasm male sterility donor CMS-234A. The second sub-cluster consisted of only KBSH-42 and the third sub-cluster consisted of KBSH-44 and KBSH-53. The second main cluster was further segregated in to five sub-clusters. The first sub-cluster grouped CMS-234 A and CMS-234B, the second sub-cluster grouped CMS 851A and CMS- 
851B, the third sub-group consisted of CMS-17A and CMS-17B, the fourth group consisted of CMS-53A and CMS-53B and the fifth group consisted of restore lines RHA 6D-1 and RHA 95-C-1. All the male sterile lines and maintainer lines studied were closely related and their genetic distances was very less $(<5)$ and similarly the linkage distances between restorer lines was eleven.

Among the five hybrids studied, hybrids KBSH-44 and KBSH-53 could be distinguishable from their parental lines using a specific SSR marker. Based on the complementary banding patterns between the hybrids and their parents, the SSR marker ORS 309 and ORS 170 was identified as the two specific markers distinguish $\mathrm{F}_{1}$ hybrid KBSH-44 form their parental lines (Figure 2).
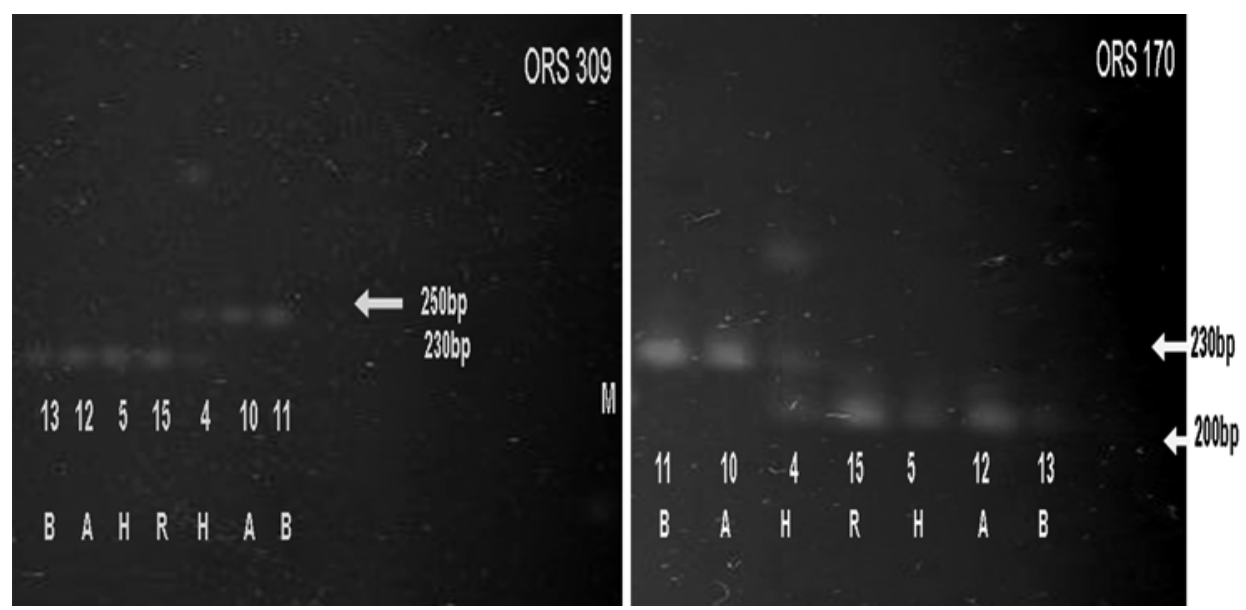

Figure 2: SSR marker ORS 309 and ORS 170 profile confirming hybridity of sunflower hybrid KBSH-44 obtained on silver stained PAGE (6\%)

KBSH-44 (4): CMS -17 A (10) $\times$ RHA 95-C-1(15);

KBSH-53 (5): CMS -53A (12) × RHA 95-C-1 (15) M: 100bp ladder

The ORS 309 amplified a specific allele of size 250 bp in $\mathrm{F}_{1}$ hybrid, seed parent (CMS-17A) and its maintainer line (CMS-17B), but not its pollen parent (RHA 95-C1). Further, the ORS 309 also amplified allele of size $230 \mathrm{bp}$ in pollen parent, RHA 95-C-1 which restores the fertility in male sterile parent. The same allele size of 230 bp was expressed in $F_{1}$ hybrid, but not in its female parent (CMS-17A). Thus, it confirmed that the allele of size $230 \mathrm{bp}$ is very specific to the pollen parent of KBSH-44 and therefore, the presence of both female and male parent alleles noticed was the result of crossing between two parents ( $F_{1}$ hybrid). The appeared banding pattern is highly specific to hybrid KBSH-44 and was not observed in any other tested hybrids. Similarly ORS 170 maker had amplified allele of size $230 \mathrm{bp}$ in female parent (CMS-17A) and maintainer line (CMS-17B), and it was absent in pollen parent (RHA 95-C-1). On the other hand the pollen parent had an amplicon at $200 \mathrm{bp}$ which was absent in female parent. However, $\mathrm{F}_{1}$ hybrid exhibited the alleles of both parents confirming the heterozygosity of the hybrid by having two bands at 
200 and $230 \mathrm{bp}$. The identified SSR in $\mathrm{F}_{1}$ hybrids showed complementary banding pattern of both the parents and found vital to distinguish the $F_{1}$ from their male and female parents.

Similarly, the newly released hybrid of sunflower KBSH-53, could be identified and distinguished by the SSR marker ORS 811 (Figure 3). The hybrid showed complementary banding pattern of both parents. The marker had amplicon of $270 \mathrm{bp}$ in its female parent (CMS 53A) and its maintainer line. The same marker had another amplicon of $230 \mathrm{bp}$ in pollen parent (RHA $95 \mathrm{C}-1$ ). The banding pattern of this hybrid showed both the amplicon at 270 and $230 \mathrm{bp}$. Thus it confirmed the genuine crossing and heterozygotic condition of the hybrid. The identified markers were further confirmed in denatured polyacrylamide vertical gel (5\%) of electrophoresis. The banding pattern was similar to that of polyacrylamide (5\%) horizontal electrophoresis.

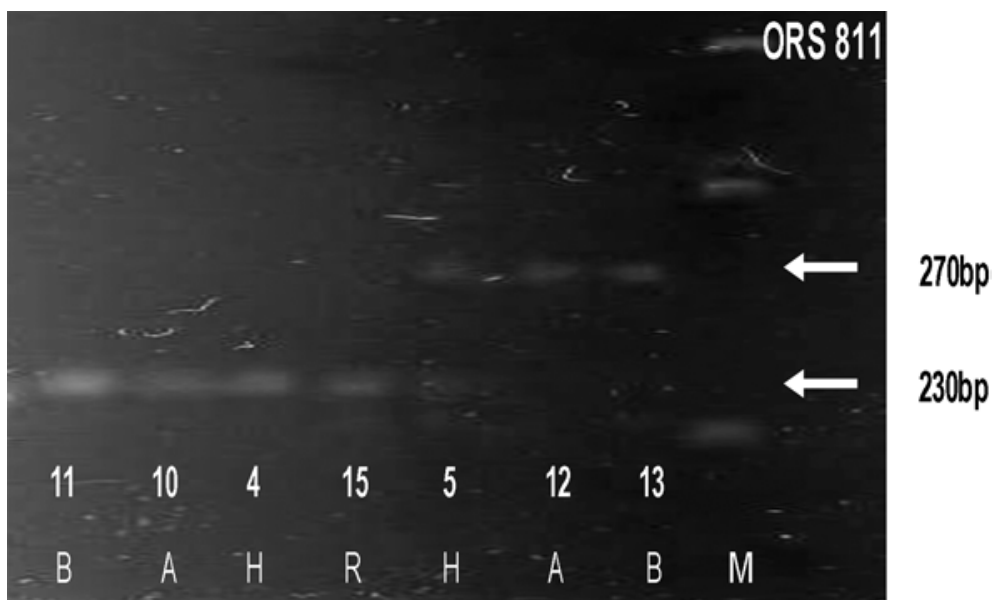

Figure 3: SSR marker ORS 811 profile confirming hybridity of sunflower hybrid KBSH-53 obtained on Ethidium bromide stained PAGE (6\%)

KBSH-44 (4): CMS-17A (10) $\times$ RHA 95-C-1(15);

KBSH-53 (5): CMS-53A (12) × RHA 95-C-1 (15) M: 100 bp ladder

The SSR markers identified had both female and male specific bands and are useful in genetic purity testing. These markers have an advantage of co-dominance inheritance, easy scoring of the alleles, reproducibility and accessibility to laboratories (Paniego et al., 2002). The use of SSR markers for genetic purity testing has also been demonstrated in maize (Wang et al., 2002), in rice (Nandakumar et al., 2004) and in cotton (Ashok and Vilas, 2005).

A cytoplasm male sterile ( $\mathrm{cms}$ ) system is being commercially exploited for hybrid seed production in sunflower as it eliminates the need for hand emasculation. $\mathrm{Cms}$ is a maternally inherited plant trait characterized by inability of flowers to produce viable pollen without affecting the female fertility and it is often associated with mitochondrial DNA rearrangement, mutation, and editing. The $\mathrm{cms}$ lines 
were multiplied with adequate isolation distance leaving no scope for a biological contamination through alien pollens coming from the neighbouring sunflower fields. Under such circumstances, the only impurity that can be expected in $\mathrm{cms}$ line seed lot that comes from its maintainer line which can probably be a mechanical admixture during various stages of $\mathrm{cms}$ lines seed handling.

An attempt was made to distinguish $\mathrm{cms}$ lines (male sterile) used for the hybrid seed production from its maintainer line (male fertile) using SSRs. The DNA samples of $\mathrm{cms}$ lines and their maintainer lines were amplified with primer pairs and resolved in polyacrylamide (6\%). Although the primers pairs studied did not show good polymorphism between "A lines" and "B lines", the SSR marker Ha 1167 showed polymorphism between different $\mathrm{cms}$ lines used for the development of different $F_{1}$ hybrids. In sunflower, the cytoplasmic male sterility is caused by a mutation in the mitochondrial genome (mtDNA) and male sterile and male fertile sunflower lines differ only in $17 \mathrm{kbp}$ fragment (Korell et al., 1992). This resulted in iso-genetic lines which differs only in one gene for pollen fertility. The gene responsible for pollen fertility is present in the mitochondrial genome and needs to be isolated for distinguishing male fertile and male sterile line. In the present study the banding pattern obtained was not so appreciable for distinguishing the sterile lines and maintainer lines. Similar results have been reported by Begona et al. (2005).

\section{CONCLUSION}

In conclusion, sunflower hybrids could be distinguishable clearly from their parental lines using SSR markers. ORS 309 and ORS 170 can be confidently used for identification of KBSH-44, but the SSR marker ORS 811 can be used for identification as well as for seed genetic purity test of KBSH-53. The study also suggests that there is a need to identify an efficient SSR or any other markers to distinguish other hybrids and $\mathrm{cms}$ lines.

\section{ACKNOWLEDGEMENT:}

Our sincere thanks to Krick House Trust, UK, funded Molecular Laboratory, University of Agricultural Sciences, Bangalore and the Scientific team for having provided the laboratory facilities and technical support for the study.

\section{REFERENCES}

Antonova, T.S., Guchetl, S.Z., Tchelustnikova, T.A. and Ramasanova, S.A., 2006. Development of marker system for identification and certification of sunflower lines and hybrids on the basis of SSR-analysis. Helia 29(45): 63-72.

Ashikawa, I., Kurata, N., Saji, S., Umehara, Y., and Sasaki, T., 1999. Application of restriction fragment fingerprinting with a rice microsatellite sequence to assembling rice YAC clones. Genome 42: 330-337. 
Dallas, J.F., 1988. Detection of DNA "fingerprints" of cultivated rice by hybridization with a human minisatellite DNA probe. Proc. Nad. Acad. Sci. USA, 85: 6831-6835.

Dongre, A. and Parkhi, P., 2005. Identification of Cotton Hybrid through the Combination of PCR Based RAPD, ISSR and Microsatellite Markers. Journal of Plant Biochem. \& Biotechnology 14: 53-55.

Korell, M., Mosges, G. and Friedt, W., 1992. Construction of a sunflower pedigree map. Helia 15: 7-16.

Lucchese, C., Dinelli, G., Miggiano, A. and Lovato, A., 1999. Identification of pepper (Capsicum spp.) cultivars by field and electrophoresis tests. Seed Science \& Technology 27: 37-47.

Nandakumar, N., Singh, A.K., Sharma, R.K., Mohapatra, T., Prabhu, K.V. and Zaman, F.U., 2004. Molecular fingerprinting of hybrids and assessment of genetic purity of hybrid seeds in rice using microsatellite markers. Euphytica 136: 257-264.

Paniego, N., Echaide, M., Muñoz, M., Fernandez, L., Torales, S., Faccio, P., Fuxan, I., Carrera, M., Zandomeni, R., Suárez, E.Y. and Hopp, H.E., 2002. Microsatellite isolation and characterization in sunflower (Helianthus annuus L.). Genome 45: 34-43.

Perez-Vich, B., Berry, S.T., Velasco, L., Fernandez-Martinez, J.M., Gandhi, S., Freeman, C., Heesacker, A., Knapp, S.J. and Leon, A.J., 2005. Molecular Mapping of Nuclear Male Sterility Genes in Sunflower. Crop Science 45: 1851-1857.

Solodenko, A.E., Sanalatij, A.V., Sivolap, Yu.M., 2003. Sunflower genotypes identification by means of microsatellite markers, (in Russian). Cytology and Genetic 38(2): 84-90.

Sundaram R. M., Naveenkumar, B., Biradar, S.K., Balachandran, S.M., Mishra, B., Ilyasahmed, M., Viraktamath, B.C., Ramesha, M.S. and Sarma, N.P., 2008. Identification of informative SSR markers capable of distinguishing hybrid rice parental lines and their utilization in seed purity assessment. Euphytica 163: 215-224.

Wang, J., Zhong, G.Y., Chin, E.C.L., Register, J.C., Riley, R.D., Nieber, W.S. and Smith, J.S.C., 2002. Identification of parents of $\mathrm{F}_{1}$ hybrids through SSR profiling of maternal and hybrid tissue. Euphytica 124: 29-34.

Yashitola, J., Thirumurugan, T., Sundaram, R.M., Naseerullah, M.K., Ramesha, M.S., Sarma, N.P. and Sonti, R.V., 2002. Assessment of purity of rice hybrids using microsatellite and STS markers. Crop Science 42: 1369-1373. 